\title{
An Analytical Study of GWAP-based Geospatial Tagging Systems
}

\author{
Ling-Jyh Chen, Yu-Song Syu, and Bo-Chun Wang \\ Institute of Information Science \\ Academia Sinica, Taiwan \\ $\{$ cclljj, yssyu, bcwang\}@iis.sinica.edu.tw
}

\author{
Wang-Chien Lee \\ Department of Computer Science and Engineering \\ The Pennsylvania State University \\ wlee@cse.psu.edu
}

\begin{abstract}
Geospatial tagging (geotagging) is an emerging and very promising application that can help users find a wide variety of location-specific information, and facilitate the development of future location-based services. Conventional geotagging systems share some limitations, such as the use of a two-phase operating model and the tendency to tag popular objects with simple contexts. To address these problems, geotagging systems based on the concept of 'Games with a Purpose' (GWAP) have been developed recently. In this study, we use analysis to investigate these new systems. Based on our analysis results, we design three metrics to evaluate the system performance, and develop five task assignment algorithms for a GWAP-based system. Using a comprehensive set of simulations under both synthetic and realistic mobility scenarios, we find that the Least-ThroughputFirst Assignment algorithm (LTFA) is the most effective approach because it can achieve competitive system utility, while its computational complexity remains moderate. We also find that, to improve the system utility, it is better to assign as many tasks as possible in each round. However, because players may feel annoyed if too many tasks are assigned at the same time, it is recommended that multiple tasks be assigned one by one in each round in order to achieve higher system utility.
\end{abstract}

\section{INTRODUCTION}

Recent surveys of the global mobile communications industry reported that handheld devices have consistently outsold laptop and desktop computers since 2004 [4]. Worldwide mobile telephone subscriptions reached 3.3 billion (i.e., $50 \%$ of the world's population) in 2007, and 59 countries had $100 \%$ mobile penetration in 2008 [4]. Clearly, mobile and wireless computing technologies affect every aspect of our working and living environments. Mobile handheld devices, which exploit the capabilities of wireless communications and people's mobility in their daily lives, have shown promise in a variety of advanced pervasive applications. Integrating such devices with other widely used technologies (e.g., environmental sensors and GPS receivers) could facilitate the development of future cyber-physical systems (CPS) [18], such as mobile urban sensing $[14,22]$, urban monitoring $[12,16]$, and geospatial tagging (geotagging) systems [2,3].

Among these applications, geotagging is an emerging and very promising application because it can help users find a wide variety of location-specific information, and thereby

This material is based upon work supported by the National Science Council of Taiwan under NSC Grants: NSC 98-2221-E-001-014-MY3, NSC 98-2631H-001-013, and NSC 98-2631-S-003-002. facilitate the development of advanced location-based services (LBS). For instance, in a geotagging system, a user might 'tag' an Italian restaurant with its latitude and longitude coordinates, a picture of the restaurant, and its menu; and another user might ask the LBS provider: "Are there any Italian restaurants nearby?" The provider will then search the geospatial database provided by the geotagging system.

A conventional geotagging system operates in two phases. First, users must prepare the GPS coordinates and other descriptive materials (e.g., text, images, or a video clip) of the target object; then they have to upload the information to the system's website. The process is inconvenient and therefore frustrates end users. Moreover, the geotagged items tend to cluster in hot spots, as they are provided voluntarily by the system users. Since most people are interested in popular items, conventional systems tend to favor general objects (e.g., museums and restaurants), rather than specialized ones (e.g., restaurants that allow pets). As a result, the scalability and extensibility of the systems are limited, so a solution that can properly address the above three issues is highly desirable.

Inspired by the concept of 'Games with a Purpose' (GWAP) $[28,30]$, several researchers have proposed GWAP-based geotagging systems in recent years $[6,9,11,15,23-26]$. Unlike conventional approaches, GWAP-based geotagging systems are asynchronously interactive. Moreover, they transform the geotagging process into a game that is entertaining and appealing to players. As a result, they can successfully outsource the geo-parsing and geo-coding processes, which are highly sophisticated and labor-intensive, to humans by taking advantage of people's desire to socialize and be entertained $[17,27]$. Since a mission can be initiated by any person, at any location, and in any context, GWAP-based geotagging systems are better able to provide information about less popular spots and specialized topics. In addition, because each mission can be initiated and processed on handhelds, the systems are not affected by the legacy problem of the two-phase operating model.

Existing GWAP-based geotagging approaches focus on the design, implementation, and measurement of real-world applications/systems; however, some recent studies have shown that the performance of GWAP systems can be improved significantly if they are played with strategies [10]. This finding motivates us to investigate design strategies for generic 
GWAP-based geotagging systems. To this end, we analyze the intrinsic properties of a generic GWAP-based geotagging system, and evaluate the system performance in terms of throughput and fairness under synthetic and realistic mobility scenarios.

The contribution of this work is three-fold. First, to model a generic GWAP-based geotagging system, we present a detailed analysis that can be applied to existing systems easily. Second, we develop three metrics to evaluate the performance of GWAP-based geotagging systems. Third, we design five task assignment algorithms, namely, the Random Assignment algorithm (RA), the Simple Assignment algorithm (SA), the Acceptance-Rate-First Assignment algorithm (ARFA), the Least-Throughput-First Assignment algorithm (LTFA), and the Hybrid Assignment algorithm (HA). We perform a comprehensive set of simulations to evaluate the algorithms' performances in various scenarios. Based on the results, we draw the following conclusions.

1) Although the HA scheme achieves the best performance in terms of system utility, it is not appropriate for realworld deployment because it is computation-hungry. In contrast, the LTFA is suitable, since its performance is comparable to that of the HA scheme, while its computational complexity is moderate and acceptable.

2) It is better to assign as many multiple tasks as possible in each round, and thereby maximize the system utility. However, players may feel annoyed if too many tasks are assigned at the same time in a round.

3) Therefore, to maximize the system utility, it is recommended that multiple tasks be assigned one by one in each round.

The remainder of this paper is organized as follows. Section II contains a review of related works on GWAP-based geospatial tagging systems. In Section III, we present our analysis of such systems, and discuss three evaluation metrics. In Section IV, we compare the five proposed task assignment algorithms, namely the RA, SA, ARFA, LTFA, and HA schemes. In Section V, we analyze the simulation results. We then summarize our conclusions in Section VI.

\section{BACKGROUND}

The Games With A Purpose (GWAP) genre [28, 30] is a type of Human Computation that outsources certain steps of the computational process to humans $[17,27]$. By taking advantage of people's desire to be entertained, GWAP attract people to play voluntarily, and also produce useful metadata as a by-product. The games have shown promise in solving a variety of problems, such as image annotation [29] and commonsense reasoning [20,31], which computer computation has been unable to resolve completely thus far.

Several GWAP-based geospatial tagging (geotagging) applications have been proposed in recent years $[6,9,11,15,23$ 26]. Generally, they can be grouped into two categories, non mission-based and mission-based, depending on the level of interaction among the players in a game. Non mission-based GWAP geotagging systems perform human computation by providing players with incentives to contribute geospatial tags voluntarily, without implementing the concept of missions [23-26]. For instance, the GeoTicTacToe [26] game, which is based on the traditional TicTacToe game, is used to study the synchronization problem in geospatial gaming systems. In the CityPoker [25] game, which is an adaptation of the popular card game, each player must try to improve his/her starting hand by changing cards at 15 locations in the real world. By analyzing the players' tracks, CityPoker provides researchers with a way to model the players' behavior and interpret the trajectory of a person moving in a spatial environment. The results can help spatially grounded intentional systems provide adequate services automatically according to the users' intentions. Moreover, CityExplorer, proposed by Matyas et al $[23,24]$, is a location-based variant of the popular board game Carcassonne [1], which only allows players to place tokens (followers) in predefined types of real-world locations. As a result, players are "forced" to explore the unstructured game area, which allows the system to collect specific geospatial data, such as the geographic coordinates and the classifications of real-world objects, as a by-product.

As their name suggests, mission-based GWAP geotagging systems generate 'missions' that players must solve. They provide incentives via a reward scheme that gives points to players when they contribute to the system (e.g., when they initiate or solve a task, or review a solution) $[6,9,11,15]$. For instance, Hitcher [11] is a location-based mobile game that exploits the cellular infrastructure. Specifically, in a Hitcher game, players create digital hitch hikers (with names, destinations and questions to ask other players) and drop them into their current cellular phone cell; or they pick up available hitchers from their current cell, answer their questions, carry them to new locations, and drop them again. As the hitchers pass from player to player, phone to phone and cell to cell, the system collects the meaningful space names of each cell as a byproduct. MobiMission [15] is a location-based pervasive social game in which missions are created, solved, and reviewed by players. The system does its best to assign "nearby" missions to players (e.g., Find a good cafe near the Empire State Building). If there are no nearby missions, it assigns "locationindependent" missions instead (e.g., Take a picture of a tall building). Similarly, in the Gopher game [9], missions are created, solved, and reviewed by players, but the system only assigns missions to "nearby" players. Finally, the Eyespy game [6] allows players to tag geographic locations with photos or text; or 'confirm' the locations of places that other players have tagged. As a result, Eyespy produces a collection of recognizable and locatable geographic details, which could be used for navigation and location-based applications.

Since non mission-based games follow certain rules, in this study, we regard them as special instances of mission-based systems with multiple tasks that are assigned all at once in each round. In this context, 'multiple tasks' means all the actions that players are allowed to take. 


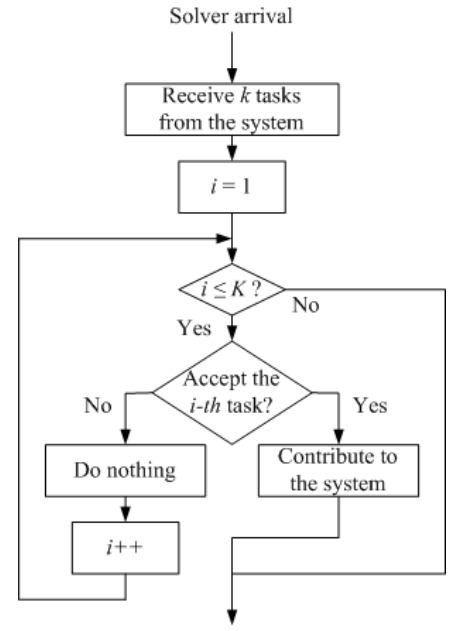

(a) all-at-once assignment

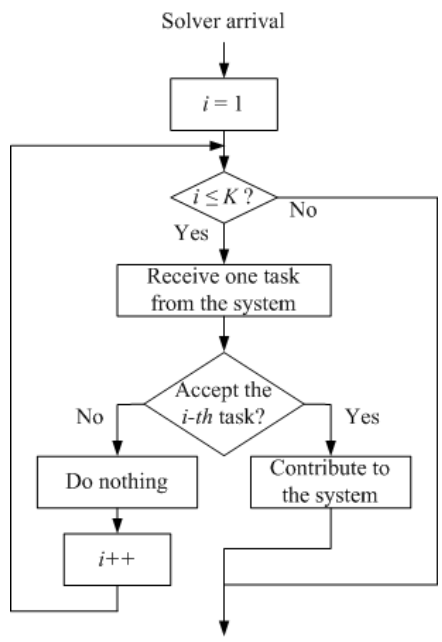

(b) one-by-one assignment
Fig. 1. The flowcharts of the task assignment approaches when a solver enters the system.

\section{GWAP-BASED GEOTAGgING SYSTEMS}

\section{A. Game Description}

In this subsection, we describe a generic GWAP-based geotagging system that is centralized and mission-based. The server is responsible for data storage and task assignment, while the clients are mobile players equipped with GPSenabled handhelds and wireless connectivity (e.g., 3G/WiFi) to the server via the Internet. There are two game-playing roles: Requester and Solver; and players are allowed to switch between the roles freely during run time.

We assume that there are $N$ locations of interest (LOI) in a game, and that the server maintains $N$ FIFO-based task queues for each LOI in the system. Let $Q_{i}$ denote the task queue of the $i$-th LOI, and let players arrive at the $i$-th LOI at a Poisson rate of $\lambda_{i}$ per time unit. We denote the ratio of the number of Requesters over the number of Solvers as $r$ (i.e., the Requester arrival rate is $\frac{r}{r+1} \lambda_{i}$ and the Solver arrival rate is $\left.\frac{1}{r+1} \lambda_{i}\right)$. The Requester always initiates a new task at his current LOI (e.g., What is the best Chinese restaurant in this area?). Thus, the task, along with the requester's ID and the timestamp, is stored in his current LOI's task queue on the server. Meanwhile, the Solver is assigned the headmost tasks of $K$ distinct task queues at most $(K<N)$ and has to decide whether to accept any one of them. The $K$ tasks may be assigned all at once, or in a one-by-one manner, as shown in Figure 1. The task acceptance decision may be based on a combination of factors, such as the distance and the popularity of the task's LOI. Once the solver accepts a task, he must solve it and thus contribute to the system.

To be effective, the system must achieve two task assignment goals. First, it must solve as many tasks (i.e., improve the assignment acceptance rate) as possible. This tends to favor popular LOIs at the expense of unpopular ones (i.e., the 'starvation' problem). Second, the system needs to strike a balance between the outcomes of LOIs (i.e., the number of each LOI's outcomes should be in proportion to the LOI's popularity). This may result in a low acceptance rate of some task assignments (i.e., the 'Equality of Outcome' problem). Thus, a clever task assignment strategy that can accommodate the two goals is highly desirable. To this end, we formulate the problem as a variant of the classic scheduling problem [7, 8, 21]. Moreover, we analyze the intrinsic properties of GWAP-based geotagging systems, and evaluate several system implementation strategies.

\section{B. Game Analysis}

First, we model the willingness of a solver $u$ to accept the assignment of a task $v$. In this study, a player's willingness is determined intuitively based on a combination of factors, such as the distance and popularity of the assigned task. Specifically, the shorter distance between $u$ and $v$, the more willing $u$ will be to accept $v$; and the more popular the associated LOI of $v$, the greater the probability that $u$ will accept $v$. We define $W(u, v)$ as the willingness (i.e., the likelihood) that $u$ will accept $v$ as follows:

$$
W(u, v)=\min \left(f_{d}(u, v) \times f_{p}(v), 0.95\right),
$$

where $f_{d}(u, v)$ represents the distance factor and $f_{p}(v)$ represents the popularity factor of the task $v$ for the solver $u$. Moreover, we set the upper bound of $W(u, v)$ at 0.95 because a player is only allowed to tackle one task in each round, even if there are multiple tasks for which the values of $f_{d}(u, v) \times f_{p}(v)$ are equal to or greater than 1.

Note that the definitions of $f_{d}(u, v)$ and $f_{p}(v)$ are flexible and depend on the implementation of the system. However, they should satisfy the following criteria: 1) the value of $f_{d}(u, v)$ decreases monotonically as the distance between $u$ 's LOI and $v$ 's LOI increases; and 2) $0 \leq f_{p}(v) \leq 1$, and the value of $f_{p}(v)$ increases monotonically with the popularity of $v$ 's LOI. We define $f_{d}(u, v)$ and $f_{p}(v)$ as follows:

- The distance factor $f_{d}(u, v)$ : We adopt the Sigmoid Function ${ }^{1}$ to model the distance factor $f_{d}(u, v)$ :

$$
f_{d}(u, v)=1.5-\frac{1}{1+e^{\tau-d(u, v)}},
$$

where $d(u, v)$ is the Euclidian distance between $u$ and $v$; $\tau$ is the threshold that determines whether the distance affects the solver's decision in a positive or negative way (i.e., $f_{d}(u, v)>1$ when the distance between $u$ and $v$ is less than $\tau$; otherwise, $f_{d}(u, v) \leq 1$, as shown in Figure 2).

- The popularity factor $f_{p}(v)$ : Since the ratio of the number of requesters over the number of solvers is $r$, the solver arrival rate at the $i$-th LOI is $\frac{\lambda_{i}}{r+1}$. Therefore, the probability that there will be $n$ solvers at the $i$-th LOI can be derived by Equation 3. The popularity factor of the task $v$ (which is located at the $i$-th LOI) is defined

\footnotetext{
${ }^{1}$ The Sigmoid Function, which starts with a small value and accelerates over time to approach a maximum, is widely used for modeling natural processes and complex system learning curves [13].
} 


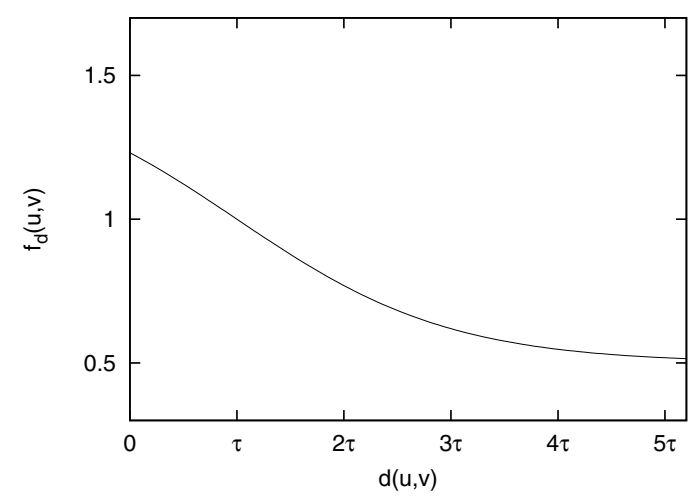

Fig. 2. Illustration of the distance factor $f_{d}(u, v)$ with different $d(u, v)$ values.

by Equation 4, i.e., the probability that there will be at least three solvers at the $i$-th LOI.

$$
\begin{gathered}
p_{i}(n)=\frac{\left(\frac{\lambda_{i}}{r+1}\right)^{n} e^{-\frac{\lambda_{i}}{r+1}}}{n !} . \\
f_{p}(v)=1-p_{i}(0)-p_{i}(1)-p_{i}(2) .
\end{gathered}
$$

However, since $\lambda_{i}$ is unknown in real systems, we use Equation 5 to approximate $\lambda_{i}$ based on the exponential moving average (EMA), where $\alpha$ is a constant smoothing factor $(0<\alpha<1)$, and $N_{i}(t)$ is the number of player arrivals at the $i$-th LOI at time $t$.

$$
\lambda_{i}^{\prime}(t+1)=\alpha \lambda_{i}^{\prime}(t)+(1-\alpha) N_{i}(t) .
$$

Let $N_{i}^{\text {Solver }}(t)$ be the number of solver arrivals at the $i$-th LOI at time $t ; u_{i}(t, j)$ be the $j$-th solver arriving the $i$-th LOI at time $t$; and $v_{i}^{k}(t, j)$ be the $k$-th task assigned to him/her $(1 \leq k \leq K)$. For ease of presentation, we use $\mathcal{W}_{k}$ to denote $W\left(u_{i}(t, j), v_{i}^{k}(t, j)\right)$. We can obtain $T^{j}(t, i)$, i.e., the expected number of the tasks completed by the $j$-th solver at the $i$-th LOI at time $t$, in two cases:

- Case 1: The headmost $K$ tasks are assigned all at once. Let $C_{k}$ be the probability that the $j$-th solver will choose to complete the $k$-th task, and $C_{0}$ be the probability that the solver will not select any of the tasks. We calculate $C_{k}$ and $C_{0}$ by Equation 6 , and then derive $T^{j}(t, i)$ by Equation 7.

$$
C_{k}=\left\{\begin{array}{l}
\mathcal{W}_{k} \prod_{l=1, l \neq k}^{K}\left(1-\mathcal{W}_{l}\right) \quad \text { if } 1 \leq k \leq K ; \\
\prod_{k=1}^{K}\left(1-\mathcal{W}_{k}\right) \quad \text { if } k=0 .
\end{array}\right.
$$

- Case 2: The headmost $K$ tasks are assigned one-by-one. In this case, $T^{j}(t, i)$ can be obtained by the complement of the probability that the $j$-th solver will decline all of the $K$ tasks, i.e.,

$$
T^{j}(t, i)=1-\prod_{k=1}^{K}\left(1-\mathcal{W}_{k}\right) .
$$

Let $T(t, i)$ be the number of tasks completed by the solver arrivals of the $i$-th LOI at time $t$; and let $A(t)$ be the overall number of tasks solved in the system up to time $t$. We derive $T(t, i)$ and $A(t)$ by Equations 9 and 10 respectively.

$$
\begin{aligned}
T(t, i) & =\sum_{j=1}^{N_{i}^{\text {Solver }}(t)} T^{j}(t, i) . \\
A(t) & =\sum_{i=1}^{N} \sum_{j=1}^{t} T(j, i) .
\end{aligned}
$$

Let $p_{i}$ denote the popularity of the $i$-th LOI. Then, we can obtain $\widetilde{T}(t, i)$, the normalized system throughput at the $i$-th LOI at time $t$ (w.r.t. $p_{i}$ ); and derive $\widetilde{\mu}(t)$, the average normalized system throughput at time $t$, by Equations 11 and 12 respectively.

$$
\begin{aligned}
& \widetilde{T}(t, i)=\frac{\sum_{j=1}^{t} T(j, i)}{p_{i}}, \\
& \widetilde{\mu}(t)=\frac{1}{N} \times \sum_{i=1}^{N} \widetilde{T}(t, i) .
\end{aligned}
$$

Moreover, the coefficient of variation of the normalized system throughput at time $t$, c.v. $(t)$, can be derived by:

$$
c . v .(t)=\frac{\sqrt{\sum_{i=1}^{N}(\widetilde{T}(t, i)-\widetilde{\mu}(t))^{2}}}{\widetilde{\mu}(t)} .
$$

\section{Evaluation Metrics}

As mentioned earlier, GWAP-based geotagging systems have two goals. To evaluate the system performance, we propose the following three evaluation metrics. First, the system prefers best-efforts, i.e., solving as many tasks as possible. Based on this criterion, we evaluate the system performance using the throughput utility metric, $U_{\text {throughput }}(t)$, which is identical to $A(t)$, i.e.,

$$
U_{\text {throughput }}(t)=A(t) .
$$

Second, the system prefers fairness, i.e., the number of solved tasks must be in proportion to the popularity of each LOI. To assess this criterion, we use the fairness utility metric, $U_{\text {fairness }}(t)$, which is the inverse of c.v. $(t)$; that is,

$$
U_{\text {fairness }}(t)=\frac{1}{c \cdot v \cdot(t)} .
$$

To accommodate the two objectives, we design the third metric called system utility, $U_{\text {system }}(t)$, by taking the product of $U_{\text {throughput }}(t)$ and $U_{\text {fairness }}(t)$, as shown in Equation 16.

$$
U_{\text {system }}(t)=U_{\text {throughput }}(t) \times U_{\text {fairness }}(t) .
$$




\section{System Strategies}

In this section, we compare five task assignment algorithms for generic GWAP-based geotagging systems, namely the Random Assignment algorithm (RA), the Simple Assignment algorithm (SA), the Acceptance-Rate-First Assignment algorithm (ARFA), the Least-Throughput-First Assignment algorithm (LTFA), and the Hybrid Assignment algorithm (HA). Let $C O P Y\left(Q_{i}\right)$ denote the function that returns a copy of the headmost task of $Q_{i}$; and let $\operatorname{POP}\left(Q_{i}\right)$ denote the function that pops up and returns the headmost task from the task queue $Q_{i}$. We discuss the five algorithms in the following sub-sections.

\section{A. Random Assignment Algorithm (RA)}

The Random Assignment Algorithm (RA) assigns the headmost tasks of $K$ distinct task queues at random. The computational complexity of the RA scheme is $O(N)$, and it provides the baseline performance of the system in this study.

\section{B. Simple Assignment Algorithm (SA)}

The Simple Assignment Algorithm (SA) assigns the $K$ headmost tasks from the current LOI's task queue. SA is the simplest scheme because its computational complexity is $O(1)$. It is adopted by most geospatial blogging systems where the requester and the solver are at the same LOI. However, as the scheme is not GWAP-based, it is not entertaining because it is short of challenges, competition, and variety [30].

\section{Acceptance-Rate-First Assignment Algorithm (ARFA)}

The Acceptance-Rate-First Assignment Algorithm (ARFA) assigns the headmost tasks of $K$ distinct task queues that are most likely to be accepted by the solver, as shown in Algorithm 1. The computational complexity of the ARFA algorithm is approximately $O(N \log N)$, since it has to sort the willingness values of the headmost tasks from the $N$ task queues. The algorithm is a greedy and best-effort approach that only tries to maximize the $U_{\text {throughput }}$ metric; therefore, it may result in the starvation problem, especially for unpopular LOIs (i.e., the willingness estimate decreases in line with the popularity factor, as shown in Equation 1).

\section{Least-Throughput-First Assignment Algorithm (LTFA)}

The Least-Throughput-First Assignment Algorithm (LTFA) assigns the headmost tasks of $K$ distinct task queues that have yielded the least throughput, as shown in Algorithm 2. For each LOI (i.e., $O(N)$ steps), the algorithm has to calculate the normalized system throughput in $O(K \lambda)$ steps, and sort the calculation results in $O(N \log N)$ steps; thus, the computational complexity of LTFA is approximately $O(N \log N+$ $N K \lambda)$. However, in practice, $N$ is far larger than $K$ and $\lambda$, so the computational complexity is approximately $O(N \log N)$. Similar to ARFA, the LTFA algorithm is a heuristics-based approach that gives priority to the $U_{\text {fairness }}$ metric, which may result in the 'equality of outcome' problem.
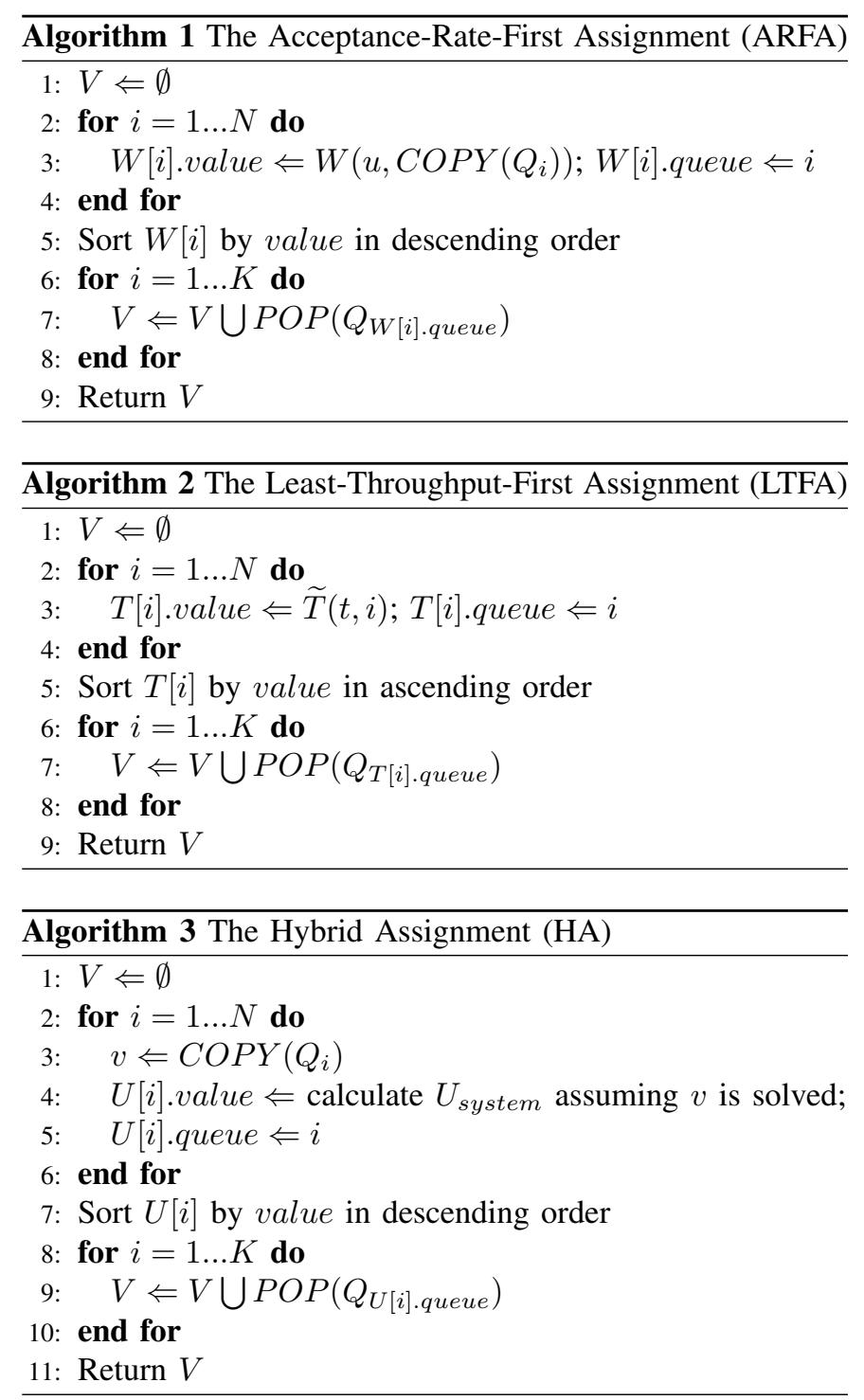

\section{E. Hybrid Assignment Algorithm (HA)}

The Hybrid Assignment Algorithm (HA) assigns the headmost task of the $K$ distinct task queues that will yield the highest system utility, as shown in Algorithm 3. For each headmost task of the $N$ queues (i.e., $O(N)$ steps), the algorithm must calculate the system utility, which is a combination of $U_{\text {throughput }}$ (i.e., $O(N)$ steps) and $U_{\text {fairness }}$ (i.e., $O(N)$ steps). Hence, the overall computational complexity of the HA algorithm is approximately $O\left(N^{2}\right)$, which is the highest among the five compared algorithms. Although this algorithm provides the optimal solution for the system, its computational overhead is a drawback.

\section{Evaluation}

We use the Monte Carlo Simulation Method to evaluate the five task assignment algorithms in three scenarios: the exponential distribution scenario (EXP), the Self-similar Least Action Walk scenario (SLAW), and the Taipei city scenario (TPE). It is assumed that there are 400 LOIs uniformly 
distributed on a $20 \times 20$ grid, and that players arrive at the $i$-th LOI at a Poisson rate of $\lambda_{i}$ per time unit. In each scenario, the value of $\lambda_{i}$ is determined as follows:

1) The EXP scenario: the value of $\lambda_{i}$ is determined based on an exponential distribution with the rate parameter set to 0.2 .

2) The SLAW scenario: we apply the SLAW model [19] to a $2,000 \times 2,000$ area, and set the SLAW parameters $n \_w p$ (i.e., the number of waypoints to be generated) and $v_{-}$Hurst (i.e., the Hurst parameter used in fractional Brownian motion) to 2,000 and 0.75 respectively. After using the SLAW generator [5] to generate the waypoints, we divide the area into a $20 \times 20$ equalsized grid, and determine the value of $\lambda_{i}$ by counting the number of waypoints in the $i$-th cell. The mean and the standard deviation of $\lambda_{i}$ are 6 and 11.4926 respectively.

3) The TPE scenario: we use the map of central Taipei (north to the Taipei SongShan Airport, east to the Taipei City Hall, south to National Taiwan University, and west to Taipei Main Station), which covers approximately 25 sq. $\mathrm{km}$, and divide it into a $20 \times 20$ equal-sized grid. To determine the value of $\lambda_{i}$, we count the number of bus stops in the $i$-th cell ${ }^{2}$. The mean and the standard deviation of $\lambda_{i}$ are 3.4485 and 2.2394 respectively.

For convenience, we set the smoothing factor $\alpha$ (cf. Equation 5) to 0.95 . Moreover, unless otherwise specified, we assume that two-thirds of the arriving players are requesters, and the other one-third comprises solvers (i.e., $r=2$ ). The distance threshold (cf. Equation 2) is set to five cell units (i.e., $\tau=5$ ). Each requester initiates a new task at his current LOI, and each solver is assigned $K$ tasks in a round using one of the five task assignment algorithms. All the results presented here are based on the average performance of 100 simulation runs.

\section{A. Evaluation results when $K=1$}

First, we evaluate the five task assignment algorithms in the three scenarios when the number of tasks assigned in each round, $K$, is equal to one. Figures 3 and 4 compare, respectively, the system throughput performance and the coefficient of variation of the normalized system throughput performance. We observe that the ARFA scheme achieves the highest throughput performance, but it yields the worst coefficient of variation performance. This is because the scheme always assigns the tasks with the highest willingness estimates (i.e., Equation 1); thus, it is more productive than the other schemes, but at the expense of increased unfairness. In contrast, because the LTFA scheme gives priority to fairness, it achieves a good coefficient of variation performance, as shown in Figure 4; however, it suffers from the equality of outcomes problem (i.e., lower throughput performance), as shown in Figure 3. The results in Figure 4 also show that the LTFA curve oscillates a great deal at the beginning of the SLAW and TPE scenarios.

\footnotetext{
${ }^{2}$ Information about bus routes in Taipei city is available at http://www.ebus.taipei.gov.tw/english/en_index_6_1.html
}

TABLE I

THE TIME REQUIRED BY EACH ALGORITHM TO FINISH ONE SIMULATION ROUND IN THE THREE SCENARIOS. (UNIT: SECONDS)

\begin{tabular}{|c|c|c|c|c|c|}
\hline & RA & SA & ARFA & LTFA & HA \\
& $O(N)$ & $O(1)$ & $O(N \log N)$ & $O(N \log N)$ & $O\left(N^{2}\right)$ \\
\hline EXP & 14.90 & 10.93 & 234.27 & 424.67 & $3,280.70$ \\
\hline SLAW & 16.07 & 11.95 & 239.68 & 460.79 & $3,230.19$ \\
\hline TPE & 13.64 & 8.95 & 85.76 & 153.13 & $1,149.50$ \\
\hline
\end{tabular}

This is because its c.v. $(t)$ performance relies to a large extent on the accuracy of the $\lambda$ estimation (cf. Equation 5), which needs a warm-up period to become stable and accurate.

By combining the two metrics, we can compare the system utility performance of the five algorithms, as shown in Figure 5. The results demonstrate that the HA scheme significantly outperforms the other schemes. We also observe that, although the RA and SA schemes represent the "golden mean" in Figures 3 and 4, they are not suitable because their system utility performance is poor. In terms of computational complexity, LTFA is the most efficient scheme (see Table I), because its system utility performance is comparable to that of the HA scheme, but its computational complexity is moderate.

Figure 6 compares the distributions of the solve rates of the five algorithms. The 'solve rate' means percentage of tasks that have been solved at each LOI. The results show that under the ARFA scheme, more than $50 \%$ of the LOIs produce zero outcomes (i.e., the solve rate is zero), which confirms our intuition that the scheme tends to suffer from the starvation problem. Moreover, we observe that the curves of the HA and LTFA schemes are nearly vertical, while the others are slanting. The results also confirm our intuition that the two schemes are better able to preserve the fairness of the normalized system throughput. In addition, the HA scheme's solve rate is higher than that of the LTFA scheme because its throughput performance is better, as shown in Figure 3. Finally, we find that the ARFA curve rises sharply in the SLAW and TPE scenarios, but not in the EXP scenario. This is because the scenarios are based on a 2D mobility model, whereas the EXP scenario is a 1D model. Consequently, the SLAW and TPE scenarios are better able to capture the spatial locality of popular LOIs. The ARFA scheme can leverage this characteristic to find a task in nearby areas easily, and thereby increase the value of the distance factor and the willingness estimate.

In addition, the results in Figure 7 show that the system utility of the SA and ARFA schemes is consistent regardless of the value of $\tau$; whereas the system utility of the RA, LTFA and HA schemes increases with $\tau$. This is because the SA scheme always assigns the tasks that are at the same LOI as the solver, and the ARFA scheme always assigns the tasks that have the highest willingness values; therefore, the two schemes are invariant to changes in the value of $\tau$. The other schemes are sensitive to the changes in $\tau$ because the willingness estimate that the solver will accept the assignment increases as $\tau$ increases (i.e., a higher $\tau$ value yields a higher distance factor value, and thus a greater willingness value). For 


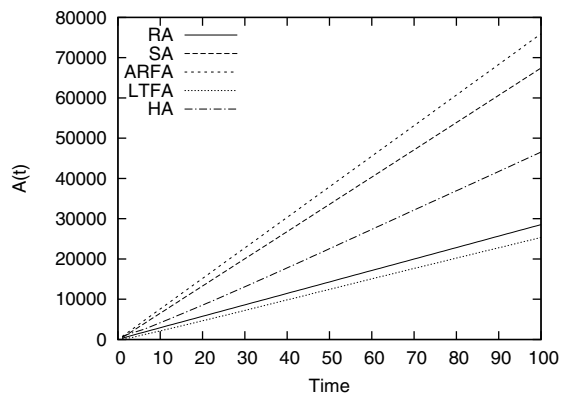

(a) EXP scenario

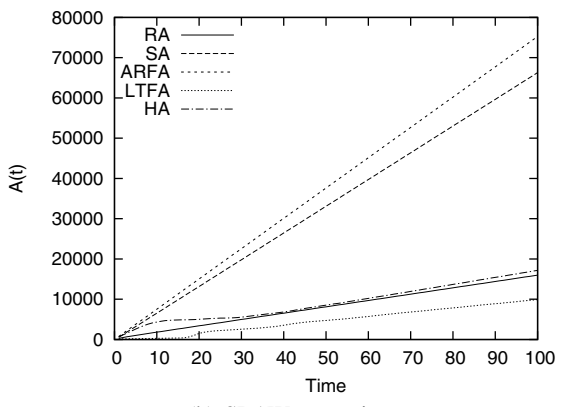

(b) SLAW scenario

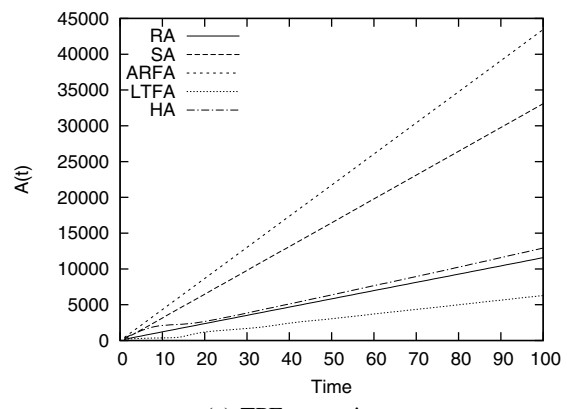

(c) TPE scenario

Fig. 3. The simulated system throughput performance in the three scenarios, when $K=1$.

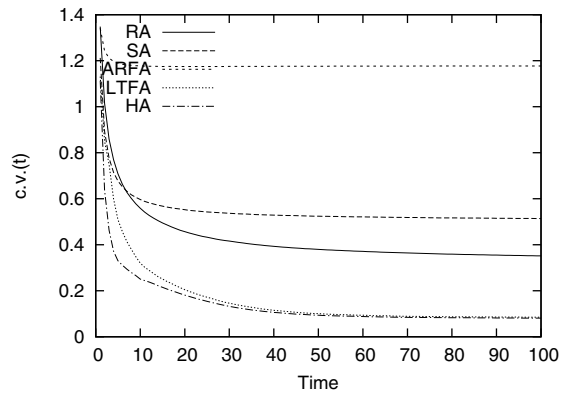

(a) EXP scenario

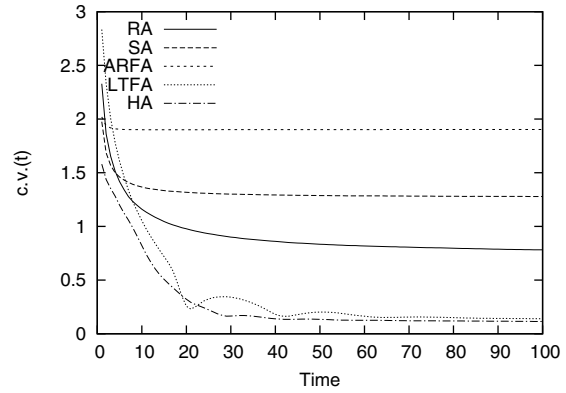

(b) SLAW scenario

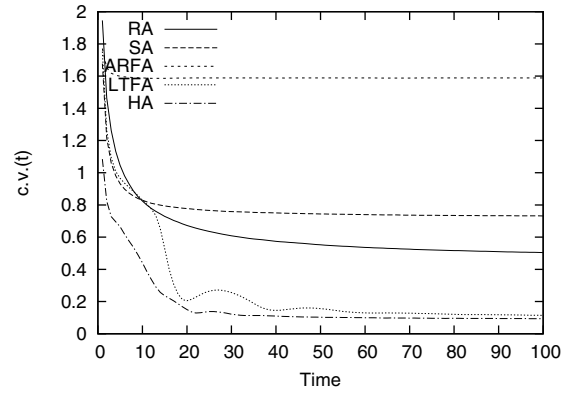

(c) TPE scenario

Fig. 4. The simulated coefficient of variation performance of the normalized system throughput in the three scenarios, when $K=1$.

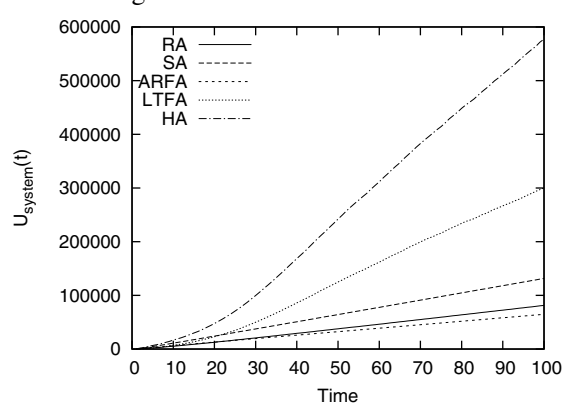

(a) EXP scenario

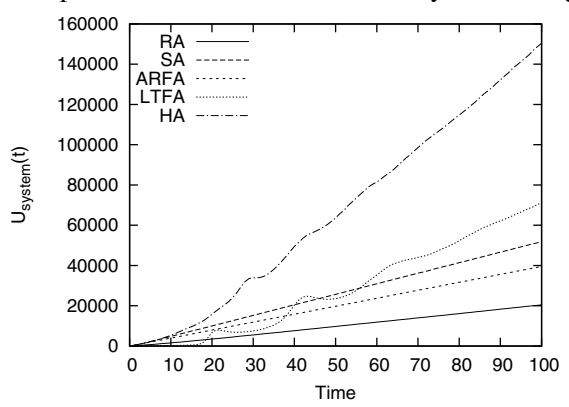

(b) SLAW scenario

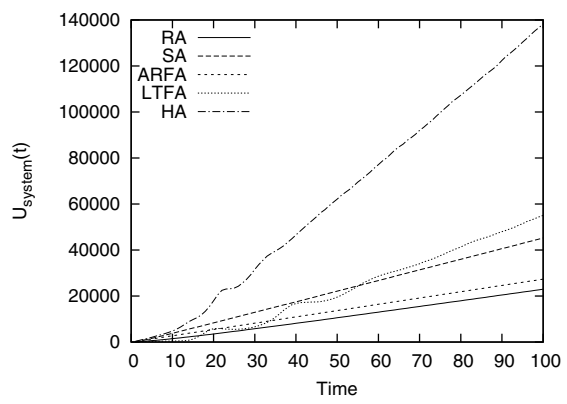

(c) TPE scenario

Fig. 5. The simulated system utility performance in the three scenarios, when $K=1$.

instance, under the LTFA scheme, the solve rate curves shown in Figure 8 shift to the right as the values of $\tau$ increase (i.e., a larger number of task assignments are accepted and solved). In each scenario, the HA scheme outperforms the other schemes, and the LTFA scheme is ranked second, regardless of the values of $\tau$.

\section{B. Evaluation results when $K>1$}

As mentioned earlier, the LTFA scheme is the most suitable for GWAP-based geotagging systems because its system utility performance is competitive, and its computational complexity is moderate. Therefore, in this subsection, we only evaluate the LTFA algorithm when the system assigns more than one task (i.e., $K>1$ ) to a solver in each game round. Figure 9 and Figure 10 show the system throughput performance and the coefficient of variation of the normalized system throughput performance when $K$ tasks are assigned in a one-by-one manner and an all-at-once manner respectively. Clearly, the system throughput increases with the value of $K$; hence, the larger the value of $K$, the faster the coefficient of variation of the normalized system throughput will converge. This is because the solver is more likely to contribute a solution to one of the assigned tasks when the value of $K$ increases, so the system throughput will increase. Moreover, since the LTFA algorithm favors the fairness criterion when assigning tasks, the coefficient of variation of the normalized system throughput performance also converges to the equilibrium state more quickly.

Figure 11 shows that the system utility increases with the value of $K$, and has a tendency to converge to an equilibrium state when $K$ is sufficiently large, regardless of whether the $K$ tasks are assigned in a one-by-one manner or an all-atonce manner. However, since the value of $K$ must not be too large in practice (i.e., otherwise, the system will annoy and discourage players), it is recommended that tasks should be assigned in a one-by-one manner, because the system utility is 


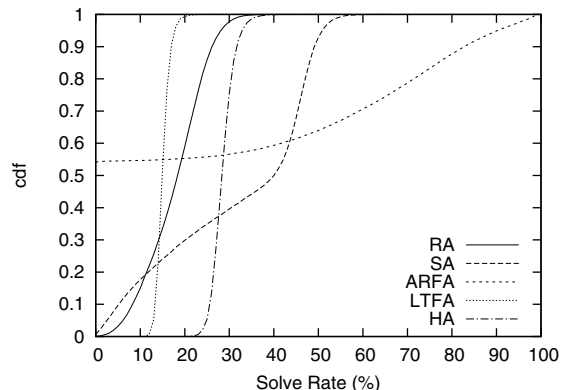

(a) EXP scenario

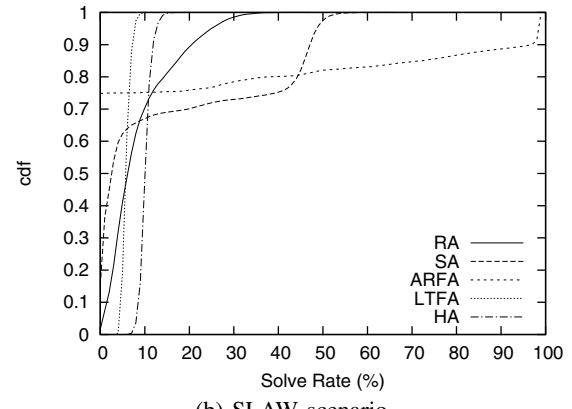

(b) SLAW scenario

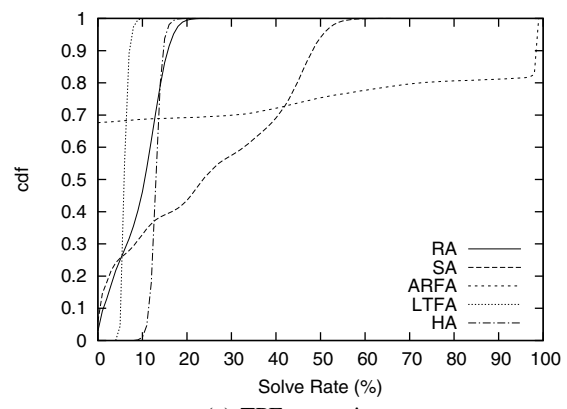

(c) TPE scenario

Fig. 6. Comparison of the CDF of the solve rate per LOI after 100 time units in the three scenarios when $K=1$.

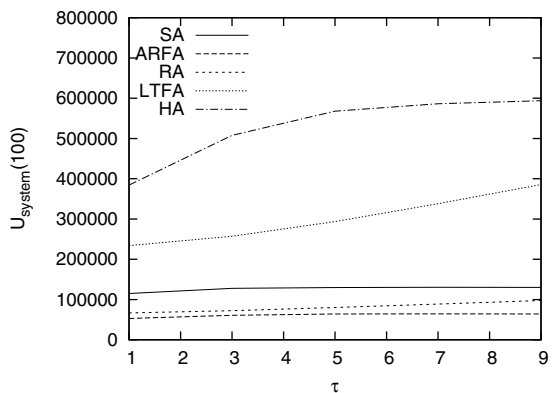

(a) EXP scenario

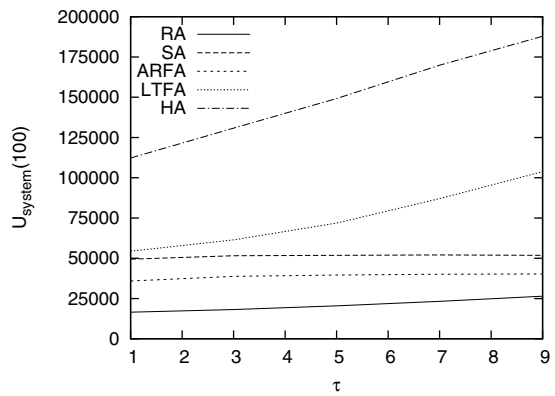

(b) SLAW scenario

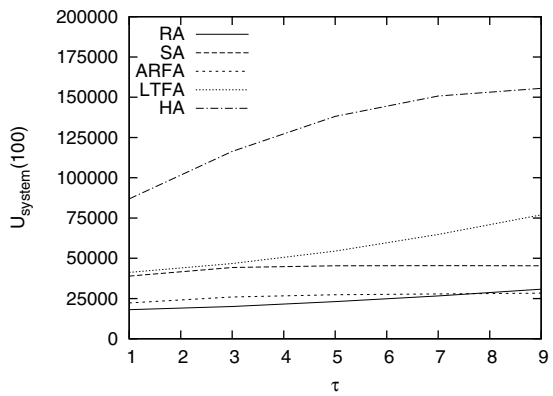

(c) TPE scenario

Fig. 7. Simulation results of the system utility performance with various $\tau$ values after 100 time units in the three scenarios when $K=1$.

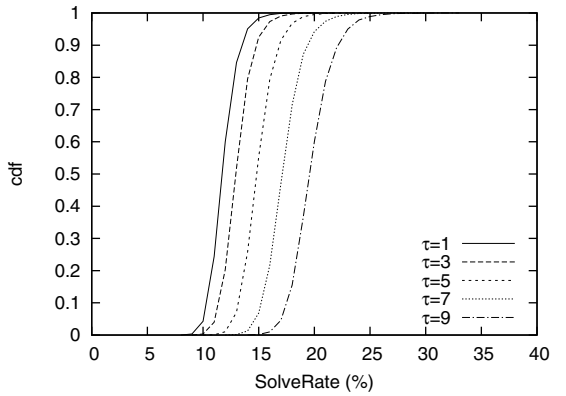

(a) EXP scenario

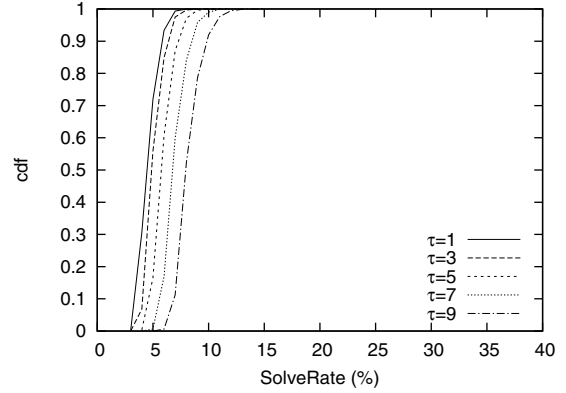

(b) SLAW scenario

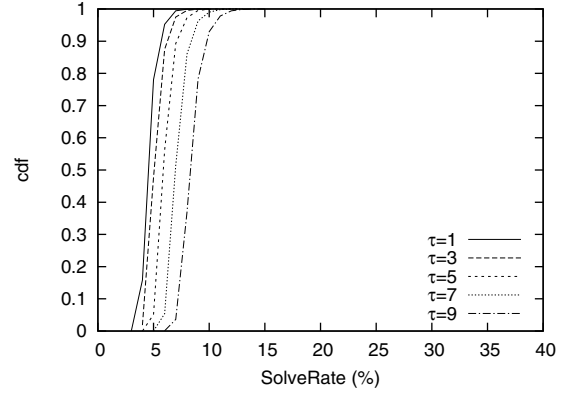

(c) TPE scenario

Fig. 8. Comparison of the CDF of the solve rate per LOI with various $\tau$ values in the three scenarios when $K=1$ and the LTFA scheme is used.

consistently higher than when tasks are assigned all at once.

\section{Discussion}

We have presented an analysis of a generic GWAP-based geotagging system, and described a comprehensive set of evaluations conducted to investigate the intrinsic properties of the system. However, some issues have yet to be addressed. In the following, we consider the issues that may arise when applying our analysis in real GWAP-based geotagging systems.

First, the proposed model only considers the throughput and fairness factors when measuring the performance of GWAPbased geotagging systems. As a result, it may not be sufficiently representative of the "productivity" and "quality" of the system. For instance, a solver may take one month to provide a high-quality solution to a task assigned by the system; or he may just provide a random outcome in five minutes. It may be possible to address this issue by adding two more factors to the model, i.e., the time required to solve a task and the quality of each solution, which can be measured by using human computation $[28,30]$. We defer a detailed evaluation of this issue to a future work.

Second, in this study, we assumed that $r=2$ in all the experiments. However, the value of $r$ depends to a large extent on the incentive mechanisms implemented in the system, so it may impact the system utility. Specifically, if $r$ is too small (i.e., most players are solvers), the system will be short of tasks to assign to solvers; conversely, if $r$ is too large (i.e., most players are requesters), the system will be short of solvers to handle tasks. Clearly, there is one 'optimal value' of $r$ that can yield the highest system utility. Hence, on the one hand, it is important to provide incentive mechanisms that can motivate as many players as possible to contribute to the system; and on the other hand, the incentive mechanism must be adjusted dynamically so that the value of $r$ can be maintained around the optimal value. The incentive system should be implemented on a case-by-case basis (i.e., it has 


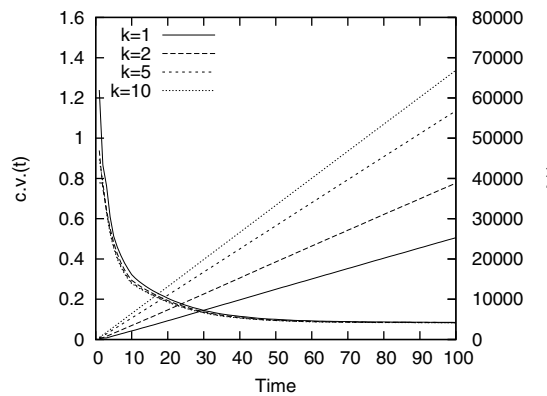

(a) EXP scenario

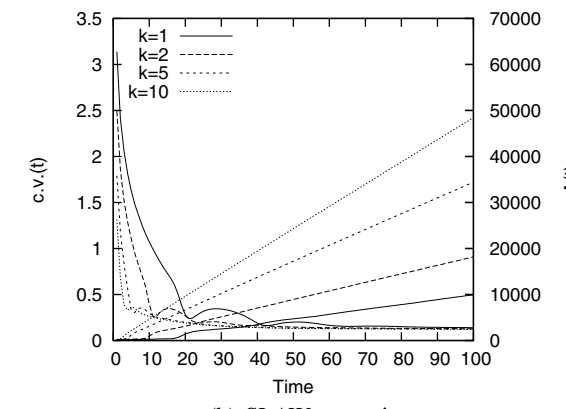

(b) SLAW scenario

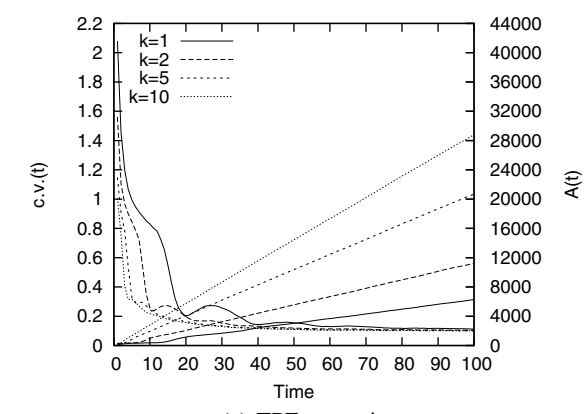

(c) TPE scenario

Fig. 9. Simulation results of the system throughput performance and the coefficient of variation performance of the normalized system throughput in the three scenarios, when the $K$ tasks are assigned by the LTFA scheme in an all-at-once manner.

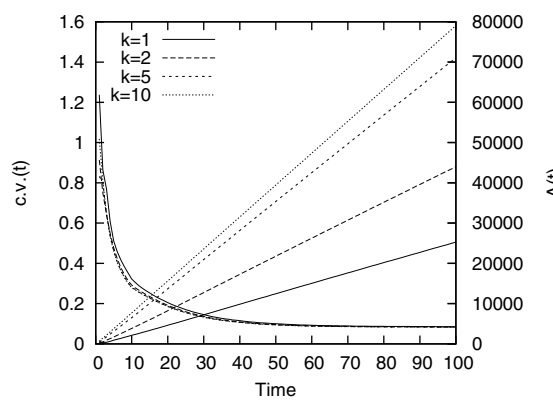

(a) EXP scenario

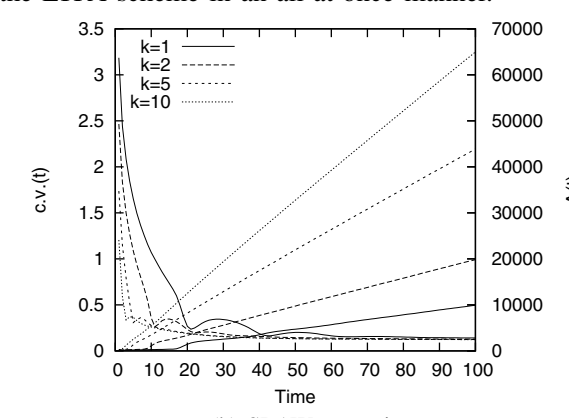

(b) SLAW scenario

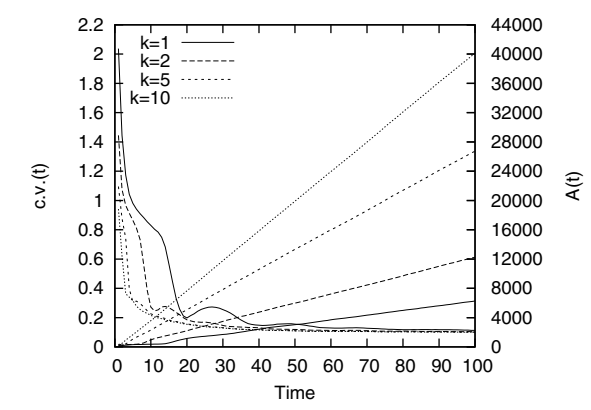

(c) TPE scenario

Fig. 10. Simulation results of the system throughput performance and the coefficient of variation performance of the normalized system throughput in the three scenarios, when the $K$ tasks are assigned by the LTFA scheme in a one-by-one manner.

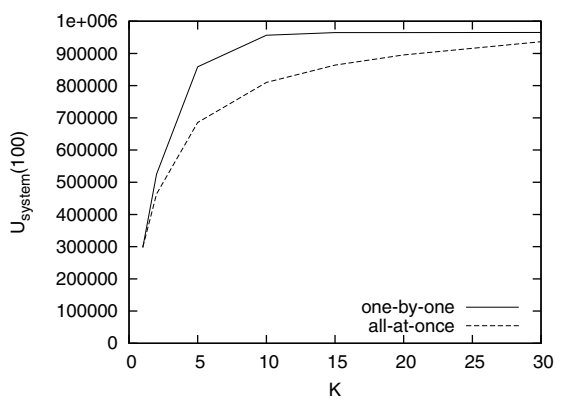

(a) EXP scenario

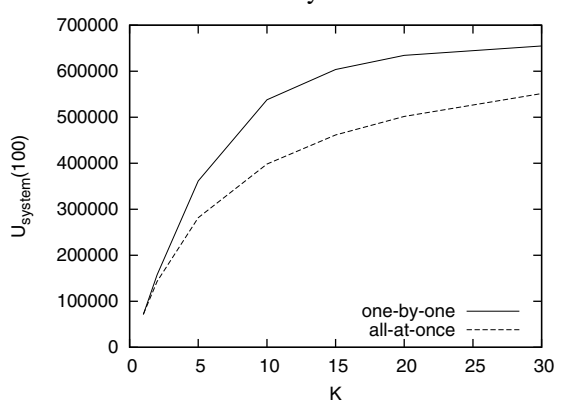

(b) SLAW scenario

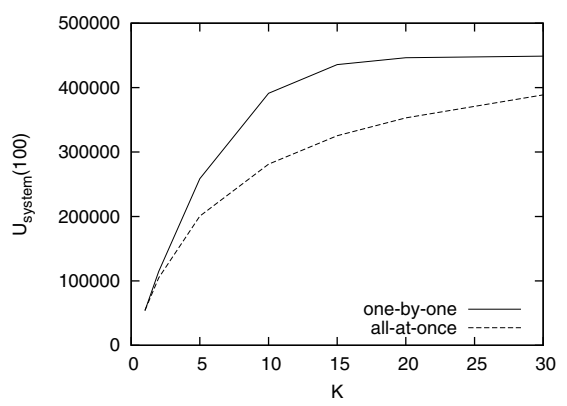

(c) TPE scenario

Fig. 11. Simulation results of the system utility performance with various values of $K$ after 100 time units, when tasks are assigned in a one-by-one manner and in an all-at-once manner respectively.

to be tailored to the specific application, the attributes of the prospective players, etc.). We also defer a detailed discussion of this issue, as well as the optimal value of $r$ with regard to the value of $K$, to a future work.

Finally, the proposed model assumes each task is a simple mission that involves only one LOI. It does not consider complex missions that comprise multiple LOIs (e.g., Find all Indian restaurants along Hollywood Boulevard) and/or the transitions among multiple LOIs (e.g., What is the fastest way to get to the airport from downtown in rush hour?). Obviously, the model requires substantial modification so that it can handle such complex missions. For example, it is necessary to 1) redefine the concept of system utility when a task is comprised of multiple LOIs; and 2) provide both quantitative and qualitative metrics to evaluate the transitions among multiple LOIs. Again, we defer a detailed discussion of this issue to a future work.

\section{CONCLUSION}

In this paper, we have studied emerging GWAP-based geotagging systems, presented an analysis of their intrinsic properties, and proposed three metrics to measure the system performance. Based on our analysis, we design five task assignment algorithms that incorporate different heuristics and optimization strategies. Using a comprehensive set of simulations of synthetic and realistic mobility scenarios, we found that the Hybrid Assignment (HA) algorithm achieves the best system utility performance, but it is too computationhungry to be deployed. In practice, the Least-Throughput-First Assignment (LTFA) algorithm is the most suitable scheme because its computational complexity is moderate, and its overall performance is comparable to that of the HA scheme. 
Moreover, to improve the system utility, it is better to assign as many tasks as possible in each game round; however, assigning too many tasks at the same time may annoy players. Finally, when multiple tasks are assigned in each round, it is better to assign them in a one-by-one manner, rather than an all-at-once manner, in order to maximize the system utility. The proposed analysis is simple and applicable to emerging GWAP-based geotagging systems. We believe the results of this study could improve the design and implementation of future GWAP-based geotagging systems.

\section{REFERENCES}

[1] Carcassonne. http://www.carcassonne.de/.

[2] GeoTagging Flickr. http://www.ickr.com/groups/geotagging/[22]

[3] Google Maps. http://maps.google.com/.

[4] IT Facts. http://www.itfacts.biz/.

[5] SLAW Generator. http://netsrv.csc.ncsu.edu/.

[6] M. Bell, S. Reeves, B. Brown, S. Sherwood, D. MacMillan, J. Ferguson, and M. Chalmers. Eyespy: Supporting navigation through play. In ACM SIGCHI, 2009.

[7] S. H. Bokhari. Assignment Problems in Parallel and Distributed Computing. Springer, 1987.

[8] T. L. Casavant and J. G. Kuhl. A taxonomy of scheduling in general-purpose distributed computing systems. IEEE Transactions on Software Engineering, 14(2):141-154, Feburary 1988.

[9] S. Casey, B. Kirman, and D. Rowland. The gopher game: a social, mobile, locative game with user generated content and peer review. In International Conference on Advances in Computer Entertainment Technology, 2007.

[10] L.-J. Chen, B.-C. Wang, and K.-T. Chen. Playing gwap with strategies. Technical report, TR-IIS-09-001, Institute of Information Science, Academia Sinica, 2009.

[11] A. Drozd, S. Benford, N. Tandavanitj, M. Wright, and A. Chamberlain. Hitchers: Designing for Cellular Positioning. In UbiComp, 2006.

[12] J. Froehlich, J. Neumann, and N. Oliver. Measuring the Pulse of the City through Shared Bicycle Programs. In ACM UrbanSensing, 2008.

[13] N. Gershenfeld. The Nature of Mathematical Modeling. Cambridge University Press, 1998.

[14] J. Goldman, K. Shilton, J. Burke, D. Estrin, M. Hansen, N. Ramanathan, S. Reddy, V. Samanta, M. Srivastava, and R. West. Participatory Sensing: A Citizen-powered Approach to Illuminating the Patterns That Shape our World. Foresight \& Governance Project, White Paper, 2009.

[15] L. Grant, H. Daanen, S. Benford, A. Hampshire, A. Drozd, and C. Greenhalgh. MobiMissions: the game of missions for mobile phones. In ACM SIGGRAPH, 2007.

[16] T. Horanont and R. Shibasaki. An Implementation of Mobile Sensing For Large-Scale Urban Monitoring. In ACM UrbanSensing, 2008.

[17] J. Howe. The Rise of Crowdsourcing. WIRED Magazine, 14(6), June 2006.
[18] E. A. Lee. Cyber physical systems: Design challenges. Technical Report UCB/EECS-2008-8, EECS Department, University of California, Berkeley, Jan 2008.

[19] K. Lee, S. Hong, S. J. Kim, I. Rhee, and S. Chong. SLAW: A Mobility Model for Human Walks. In IEEE Infocom, 2009.

[20] H. Lieberman, D. Smith, and A. Teeters. Common Consensus: a web-based game for collecting commonsense goals. In ACM Workshop on Common Sense for Intelligent Interfaces, 2007.

[21] V. M. Lo. Heuristic algorithms for task assignment in distributed systems. IEEE Transactions on Computers, 37(11):1384-1397, November 1988.

H. Lu, W. Pan, N. D. Lane, T. Choudhury, and A. T. Campbell. SoundSense: Scalable Sound Sensing for People-Centric Sensing Applications on Mobile Phones. In ACM/USENIX MobiSys, 2009.

[23] S. Matyas. Playful geospatial data acquisition by location-based gaming communities. The International Journal of Virtual Reality, 6(3):1-10, 2007.

[24] S. Matyas, C. Matyas, C. Schlieder, P. Kiefer, H. Mitarai, and M. Kamata. Designing Location-based Mobile Games With A Purpose: Collecting Geospatial Data with CityExplorer. In ACM ACE, 2008.

[25] C. Schlieder. Representing the Meaning of Spatial Behavior by Spatially Grounded Intentional Systems. Lecture Notes in Computer Science, 3799:30-44, 2005.

[26] C. Schlieder, P. Kiefer, and S. Matyas. Geogames: Designing Location-Based Games from Classic Board Games. IEEE Intelligent Systems, 21(5):40-46, 2006.

[27] J. Surowiecki. The Wisdom of Crowds: Why the Many Are Smarter Than the Few and How Collective Wisdom Shapes Business, Economies, Societies and Nations. Doubleday, May 2004.

[28] L. von Ahn. Games with a Purpose. IEEE Computer, 39(6):92-94, June 2006.

[29] L. von Ahn and L. Dabbish. Labeling Images with a Computer Game. In ACM SIGCHI, 2004.

[30] L. von Ahn and L. Dabbish. Designing games with a purpose. Communications of the ACM, 51(8):58-67, August 2008.

[31] L. von Ahn, M. Kedia, and M. Blum. Verbosity: A Game for Collecting Common-Sense Facts. In ACM SIGCHI, 2006. 